\title{
Association between the cardiometabolic index and non-alcoholic fatty liver disease: insights from a general population
}

\author{
Jiawang Zou ${ }^{1}$, Hailan Xiong ${ }^{1}$, Huimin Zhang ${ }^{2}$, Chong Hu${ }^{3}$, Song Lu ${ }^{2}$ and Yang Zou ${ }^{4}$
}

\begin{abstract}
Background: Emerging evidence suggests that cardiometabolic index (CMI) is closely related to diabetes, hypertension, stroke, cardiovascular disease, and kidney disease, which implies that CMI has the value as an indicator of metabolic diseases. However, data on the relationships between CMI and non-alcoholic fatty liver disease (NAFLD) risks have not been reported. This study is designed to examine the association between CMI and NAFLD in the general population.

Methods: The current study included 14,251 subjects whose CMI was the product of triglyceride/high-density lipoprotein cholesterol ratio and waist-to-height ratio. Linear regression was used to analyze the correlation between baseline information and CMI, logistic regression was used to study the relationship between CMI and NAFLD, and subgroup analysis was used to explore potential high-risk groups.

Results: After adjusted for potential confounding factors, higher CMI was independently associated with NAFLD, in which every additional standard deviation (SD) of CMI increased the risk of NAFLD by 28\% (OR 1.28 per SD increase, $95 \% \mathrm{Cl}$ 1.19-1.37, $P$ for trend < 0.0001). There were also significant differences in CMI-related NAFLD risk among different ages and genders, in which the CMI-related NAFLD risk in young people was significantly higher than that in other age groups ( $O R=2.63$ per SD increase for young people, $\mathrm{OR}=1.38$ per SD increase for young and middle-aged people, $\mathrm{OR}=1.18$ per SD increase for middle-aged and elderly people; $\mathrm{OR}=1.14$ per SD increase for elderly people, $P$ for interaction $=0.0010$ ), and the CMI-related NAFLD risk in women was significantly higher than that in men $(\mathrm{OR}=1.58$ per SD increase for women, $\mathrm{OR}=1.26$ per SD increase for men, $P$ for interaction $=0.0045)$.
\end{abstract}

Conclusions: Current studies have found that after excluding potential confounding factors, higher CMI in the general population is independently associated with NAFLD risk.

Keywords: Cardiometabolic index, Non-alcoholic fatty liver disease, General population, CMI, NAFLD

\section{Background}

Non-alcoholic fatty liver disease (NAFLD) has escalated to a major public health problem worldwide [1, 2]. Similar to several other chronic diseases, NAFLD is extremely common in the general population. Recent

\footnotetext{
*Correspondence: jxyxyzy@163.com

${ }^{4}$ Jiangxi Cardiovascular Research Institute, Jiangxi Provincial People's Hospital, Nanchang 330006, China

Full list of author information is available at the end of the article
}

epidemiological surveys indicate that about a quarter of adults worldwide suffer from NAFLD [3], and with the popularity of diabetes and obesity, diet and lifestyle changes, the aging of the population intensified, the prevalence of NAFLD is still constantly increasing. It is expected that in 10 years, the total number of NAFLD patients in the world will reach $1 / 3$ of the total population, which will bring an inestimable disease burden to the whole mankind [3-6]. Furthermore, NAFLD is not 
benign static liver disease. Many studies have shown that NAFLD is a systemic, multi-system disease with continuous progression [7, 8]; It will affect the metabolic status of the body and act on the heart, brain, kidney, peripheral blood vessels and other target organs, resulting in adverse health consequences [8-10]. Therefore, it would be meaningful to identify the risk factors for NAFLD at an early stage.

The association of dyslipidemia, obesity and NAFLD has been well documented [11, 12]. Among them, the lipid ratio of triglyceride/high-density lipoprotein cholesterol (TG/HDL-C) is considered to be an excellent parameter for predicting the risk of NAFLD, which can better assess insulin resistance (IR) in the NAFLD population [13-15]. Waist-to-height ratio (WHtR) is a combination of waist circumference (WC) and height. Compared with the traditional simple body measurement index, it can better identify abdominal obesity, evaluate NAFLD, cardiac metabolic risk and a variety of noncommunicable diseases [16-19]. More recently, Wakabayashi et al. developed a marker called cardiometabolic index (CMI) [20], calculated as WHtR multiplied by TG/ HDL-C ratio, which combines lipid and obesity parameters into a simple and repeatable marker for the effective identification of diabetes [20,21]. Additionally, several recent studies have found that CMI is closely related to hypertension, stroke, cardiovascular disease and kidney disease [22-25]. These results imply that CMI is significant as an indicator of metabolic diseases. However, research on the potential relationships between CMI and NAFLD risks has not been reported. This study is designed to examine the association between CMI and NAFLD in the general population.

\section{Methods}

\section{Research design and data sources}

NAGALA is a cohort study based on the general population, which has been going on since 1994. It aims to evaluate the risk factors of common chronic diseases in the general population, and provides useful research data for the early prevention and treatment of chronic diseases. The design scheme of NAGALA has been described in detail previously [26]. This study was a post hoc analysis of NAGALA, and a cross-sectional design was adopted according to the new research hypothesis, in which the available research data have been uploaded to the DRYAD database by Hamaguchi et al. [27]. This study extracted data from a general population of 20,944 people recruited in the NAGALA program who took a comprehensive health examination in Murakami Memorial Hospital from 2004 to 2015. According to the purpose of the study, subjects with alcoholic/viral hepatitis, diabetes, impaired fasting blood glucose, incomplete covariates and excessive drinking were excluded, and 14,251 subjects were included in the final analysis of this study. Since NAGALA's research scheme has been previously approved by the Murakami Memorial Hospital institutional review committee (IRB number: 2018-09-01), and the subject's identification information has been replaced with a check code, there is no needless for separate ethical approval and informed consent in this study.

\section{Clinical characteristics and biochemical parameters}

Clinical characteristic parameters such as sex, height, weight, WC, age, smoking/drinking status, systolic/diastolic blood pressure (S/DBP) and habit of exercise were evaluated and recorded by trained medical workers with a standard questionnaire. The subjects were classified according to their weekly alcohol consumption and smoking status, and the drinking status was divided into three categories: non or less drinking $(<40 \mathrm{~g} / \mathrm{w})$, light drinking (40-139 g/w) and moderate drinking $(140-209 \mathrm{~g} / \mathrm{w})$ [28]. Smoking status can be divided into three categories: non-smoking, past smoking and current smoking. Habit of exercise was divided into groups according to the subjects' weekly exercise, in which regular participation in any type of exercise at least once a week was considered to have exercise habits. Hematological samples used to analyze biochemical parameters were collected at least $8 \mathrm{~h}$ after fasting, and an automatic analyzer was used to analyze and determine gammaglutamyl transferase (GGT), hemoglobin A1c (HbA1c), aspartate aminotransferase (AST), HDL-C, total cholesterol (TC), TG, fasting blood glucose (FPG) and alanine aminotransferase (ALT) according to the standard method.

\section{Diagnosis of NAFLD}

As previously described [26], the diagnosis of NAFLD in the NAGALA project was carried out by ultrasound. Ultrasound examinations were performed by professional technicians, and ultrasound images were evaluated by experienced gastroenterologists without knowing the subjects' other examination and diagnostic information, based on the echo of the hepatorenal echo contrast, liver brightness, deep attenuation and vascular fuzzy four criteria for the score of 0 to 4 points, a comprehensive score greater than 2 points in the diagnosis of NAFLD [29].

\section{Statistical analysis}

The statistical software Empower Stats (version 2.0) and $\mathrm{R}$ language (version 3.4.3) were used for data analysis. To further understand the correlation between CMI and NAFLD, this study took CMI as a continuous variable and a categorical variable for correlation analysis. The baseline characteristics of all patients were stratified 
by CMI quartile, and categorical variables and continuous variables were described as percentages or mean (standard deviation: SD) or median (interquartile range) according to the type and distribution pattern of variables. Chi-square test or one-way ANOVA or rank-sum test were used for inter-group comparison, and linear regression was used to check the association between baseline variables and CMI. Univariate and multivariate logistic regression models were then used to determine whether CMI was independently associated with NAFLD, and the results show that the odds ratio (OR) and the corresponding 95\% confidence interval (CI). In the multivariate regression analysis, we ran a total of three models. According to the STROBE statement [30], we adopted different variable adjustment strategies in the three models. Model one, as a fine-tuning model, only adjusted the non-collinear general demographic variables (Additional file 1: Table S1), including sex, age, body mass index (BMI), drinking status and smoking status. In model two, the non-collinear covariates (that affecting the matching risk of CMI and NAFLD by more than $10 \%$ were regarded as confounding variables) were adjusted [31]. Model three further adjusted the non-collinear covariates that were statistically related to NAFLD in univariate analysis based on Model two. Additionally, we also conducted an exploratory stratified analysis among people of different ages, genders, BMI and whether or not to preserve exercise habits, and checked whether the effect of CMI on NAFLD was different among different subgroups by the likelihood ratio test. The receiver operating characteristic (ROC) curve was constructed to evaluate the accuracy of WC, height, TG, HDL-C and CMI in determining NAFLD, and the best cut-off value of CMI was calculated. In addition, we further used restricted cubic splines to flexibly model the association between the continuous variable CMI and NAFLD.

\section{Results}

\section{Subject characteristics}

Overall 14,251 subjects were enrolled in this study, and the study population was quartered according to the CMI quartile. Table 1 summarizes the clinical and biochemical characteristics of the study population. The prevalence rates of NAFLD in the four groups were 1.26\%, 5.96\%, $17.56 \%$ and $45.52 \%$, respectively. There were significant differences in parameters and indicators among the CMI groups (all $P<0.0001$ ). Compared with the group with low CMI, the group with high CMI was older, had more people with smoking and drinking status, and had higher anthropometric parameters such as height, weight, BMI, WC and blood pressure, while the serological parameters except HDL-C increased gradually with the increase of CMI.

\section{Correlation analysis between $\mathrm{CMI}$ and baseline variables}

The correlation between CMI and baseline variables was evaluated using linear regression, and the results are summarized in Table 2. It is obvious from the table that all the baseline variables have a linear correlation with CMI, in which habit of exercise, HDL-C has a negative correlation with CMI, while other variables have a positive correlation with CMI. It is worth noting that the related variables of blood glucose and blood lipid metabolism were strongly correlated with CMI, and the correlation between WHtR and CMI is the strongest. This finding hints at a possibility that CMI has the value as an index of metabolic diseases; Additionally, these variables significantly correlated with CMI may be cofactors for the association between CMI and NAFLD.

\section{Association between CMI and NAFLD}

Table 3 shows the results of univariate logical regression analysis. In univariate analysis, except for drinking status, all the covariables were significantly correlated with NAFLD, and the correlation between CMI and NAFLD was the highest (OR 19.64, 95\% CI 16.97-22.73); These covariates related to NAFLD will be incorporated into the multivariable model as confounding factors. In order to be consistent with the results of previous studies [22-25], CMI was also converted into $\mathrm{Z}$ scores for analysis in this study. In the multivariate regression analysis (Table 4), after adjusting the general demographic variables, model one found that CMI was positively correlated with NAFLD (OR 1.67 per SD increase, 95\% CI 1.58-1.77), and the NAFLD risk gradually increased among the CMI quartiles [OR (Q1:1, Q2:4.95, Q3:16.64, Q4:65.29, $P$ for trend $<0.0001)$. Model two adjusted for non-collinear covariates that altered the matching risk of CMI and NAFLD by more than $10 \%$, the positive correlation weakened slightly (OR 1.28 per SD increase, $95 \% \mathrm{CI}$ $1.20-1.37, P$ for trend $<0.0001)$. After further adjusting the non-collinear covariates related to NAFLD in univariate analysis (Model three: OR 1.28 per SD increase, 95\% CI 1.19-1.37, $P$ for trend $<0.0001$ ), the positive correlation between them remained stable. In short, CMI is an independent risk factor for NAFLD.

\section{Subgroup analysis}

In the subgroup analysis, the researchers further evaluated whether CMI and NAFLD differed between different populations by stratification analysis and interactivity tests. The age and BMI were stratified according to the commonly used clinical cutoff points, and the results 
Table 1 Characteristics of the subject

\begin{tabular}{|c|c|c|c|c|c|}
\hline & CMI quartile & & & & $P$-value \\
\hline & Q1 (0.01-0.13) & Q2 (0.13-0.23) & Q3 (0.23-0.43) & Q4 (0.43-7.99) & \\
\hline No. of subjects & 3561 & 3560 & 3560 & 3561 & \\
\hline Age, years & $40.00(35.00,46.00)$ & $42.00(36.00,49.00)$ & $44.00(38.00,51.00)$ & $45.00(39.00,52.00)$ & $<0.001$ \\
\hline Sex & & & & & $<0.001$ \\
\hline Women & 2792 (78.40\%) & 2094 (58.82\%) & $1320(37.08 \%)$ & $634(17.80 \%)$ & \\
\hline Men & 769 (21.60\%) & $1466(41.18 \%)$ & $2240(62.92 \%)$ & 2927 (82.20\%) & \\
\hline Weight, kg & $51.30(46.80,56.70)$ & $55.90(50.00,62.90)$ & $61.70(55.00,68.30)$ & $69.00(62.10,76.30)$ & $<0.001$ \\
\hline Height, cm & $161.78 \pm 7.49$ & $163.61 \pm 8.59$ & $165.66 \pm 8.52$ & $168.12 \pm 7.94$ & $<0.001$ \\
\hline $\mathrm{BMI}, \mathrm{kg} / \mathrm{m}^{2}$ & $19.95 \pm 2.12$ & $21.18 \pm 2.48$ & $22.54 \pm 2.69$ & $24.59 \pm 3.10$ & $<0.001$ \\
\hline$W C, \mathrm{~cm}$ & $69.35 \pm 6.37$ & $73.44 \pm 7.34$ & $77.87 \pm 7.50$ & $84.06 \pm 7.84$ & $<0.001$ \\
\hline NAFLD & $45(1.26 \%)$ & $212(5.96 \%)$ & $625(17.56 \%)$ & $1621(45.52 \%)$ & $<0.001$ \\
\hline $\mathrm{WHtR}$ & $0.43 \pm 0.04$ & $0.45 \pm 0.04$ & $0.47 \pm 0.04$ & $0.50 \pm 0.05$ & $<0.001$ \\
\hline$A L T, I U / L$ & $14.00(11.00,17.00)$ & $15.00(12.00,19.00)$ & $17.00(13.00,23.00)$ & $23.00(17.00,32.00)$ & $<0.001$ \\
\hline AST, IU/L & $16.00(13.00,19.00)$ & $16.00(13.00,20.00)$ & $17.00(14.00,21.00)$ & $19.00(16.00,24.00)$ & $<0.001$ \\
\hline GGT, IU/L & $12.00(10.00,15.00)$ & $13.00(10.00,17.00)$ & $16.00(12.00,22.00)$ & $21.00(15.00,31.00)$ & $<0.001$ \\
\hline $\mathrm{HDL}-\mathrm{C}, \mathrm{mmol} / \mathrm{L}$ & $1.84 \pm 0.37$ & $1.57 \pm 0.29$ & $1.35 \pm 0.25$ & $1.08 \pm 0.21$ & $<0.001$ \\
\hline $\mathrm{TC}, \mathrm{mmol} / \mathrm{L}$ & $4.85 \pm 0.81$ & $5.00 \pm 0.80$ & $5.18 \pm 0.86$ & $5.47 \pm 0.87$ & $<0.001$ \\
\hline $\mathrm{TG}, \mathrm{mmol} / \mathrm{L}$ & $0.38(0.30,0.45)$ & $0.60(0.52,0.70)$ & $0.87(0.75,1.00)$ & $1.48(1.21,1.91)$ & $<0.001$ \\
\hline TG/HDL-C ratio & $0.22(0.17,0.26)$ & $0.39(0.34,0.44)$ & $0.65(0.56,0.76)$ & $1.33(1.06,1.84)$ & $<0.001$ \\
\hline $\mathrm{FPG}, \mathrm{mmol} / \mathrm{L}$ & $4.95 \pm 0.39$ & $5.08 \pm 0.40$ & $5.21 \pm 0.38$ & $5.34 \pm 0.37$ & $<0.001$ \\
\hline $\mathrm{HbA} 1 \mathrm{c}, \%$ & $5.14 \pm 0.30$ & $5.15 \pm 0.31$ & $5.19 \pm 0.33$ & $5.24 \pm 0.34$ & $<0.001$ \\
\hline $\mathrm{SBP}, \mathrm{mmHg}$ & $107.26 \pm 12.71$ & $111.51 \pm 13.66$ & $115.81 \pm 14.41$ & $121.15 \pm 14.73$ & $<0.001$ \\
\hline $\mathrm{DBP}, \mathrm{mmHg}$ & $66.22 \pm 8.97$ & $69.25 \pm 9.51$ & $72.54 \pm 9.95$ & $76.47 \pm 10.16$ & $<0.001$ \\
\hline Habit of exercise & & & & & $<0.001$ \\
\hline No & 2886 (81.04\%) & 2941 (82.61\%) & 2934 (82.42\%) & 3013 (84.61\%) & \\
\hline Yes & 675 (18.96\%) & $619(17.39 \%)$ & $626(17.58 \%)$ & $548(15.39 \%)$ & \\
\hline Drinking status & & & & & $<0.001$ \\
\hline Nor or less & 3185 (89.44\%) & 2983 (83.79\%) & $2866(80.51 \%)$ & 2768 (77.73\%) & \\
\hline Light & 298 (8.37\%) & $438(12.30 \%)$ & 505 (14.19\%) & $513(14.41 \%)$ & \\
\hline Moderate & 78 (2.19\%) & 139 (3.90\%) & 189 (5.31\%) & $280(7.86 \%)$ & \\
\hline Smoking status & & & & & $<0.001$ \\
\hline Nor & 2881 (80.90\%) & $2437(68.46 \%)$ & $1929(54.19 \%)$ & 1495 (41.98\%) & \\
\hline Past & $388(10.90 \%)$ & $567(15.93 \%)$ & 745 (20.93\%) & $856(24.04 \%)$ & \\
\hline Current & 292 (8.20\%) & $556(15.62 \%)$ & 886 (24.89\%) & 1210 (33.98\%) & \\
\hline
\end{tabular}

Values were expressed as mean (SD) or medians (quartile interval) or $\mathrm{n}(\%)$

NAFLD, non-alcoholic fatty liver disease; BMI, body mass index; WC, Waist circumference; WHtR, waist-to-height ratio; ALT, alanine aminotransferase; AST, aspartate aminotransferase; GGT, gamma-glutamyl transferase; HDL-C, high-density lipoprotein cholesterol; TC, total cholesterol; TG, triglyceride; HbA1c, hemoglobin A1C; FPG, fasting plasma glucose; SBP, systolic blood pressure; DBP, Diastolic blood pressure; CMI, cardiometabolic index

of stratified analysis and interactive test suggested that (Table 5): there were significant differences between CMI and NAFLD in different ages and genders $(P$ for interaction $<0.05$ ), but no significant statistical differences were found in BMI and habit of exercise stratification $(P$ for interaction $>0.05$ ). Of note, this study found that the CMI-related NAFLD risk of young people was significantly higher than that of other age groups $(\mathrm{OR}=2.63$ per $\mathrm{SD}$ increase for young people, $\mathrm{OR}=1.38$ per SD increase for young and middle-aged people,
$\mathrm{OR}=1.18$ per $\mathrm{SD}$ increase for middle-aged and elderly people; $\mathrm{OR}=1.14$ per $\mathrm{SD}$ increase for elderly people, $P$ for interaction $=0.0010$ ), and the CMI-related NAFLD risk of women was significantly higher than that of men $(\mathrm{OR}=1.58$ per $\mathrm{SD}$ increase for women, $\mathrm{OR}=1.26$ per $\mathrm{SD}$ increase for men, $P$ for interaction $=0.0045$ ).

\section{Determine the best cutoff value for NAFLD}

ROC analysis was performed to calculate WC, height, TG, HDL-C and CMI for determining the area under 
Table 2 Association between CMI and baseline variables

\begin{tabular}{|c|c|c|c|}
\hline & Statistics & Effect size $(\beta)$ & $P$-value \\
\hline \multicolumn{4}{|l|}{ Sex } \\
\hline Women & $6840(48.00 \%)$ & Ref & \\
\hline Men & 7411 (52.00\%) & $0.26(0.25,0.28)$ & $<0.0001$ \\
\hline Age & $43.53 \pm 8.89$ & $0.01(0.00,0.01)$ & $<0.0001$ \\
\hline Weight & $60.26 \pm 11.61$ & $0.01(0.01,0.02)$ & $<0.0001$ \\
\hline Height & $164.80 \pm 8.48$ & $0.01(0.01,0.01)$ & $<0.0001$ \\
\hline BMI & $22.06 \pm 3.14$ & $0.06(0.05,0.06)$ & $<0.0001$ \\
\hline WC & $76.19 \pm 9.10$ & $0.02(0.02,0.02)$ & $<0.0001$ \\
\hline $\mathrm{WH} \mathrm{tR}$ & $0.46 \pm 0.05$ & $3.27(3.16,3.38)$ & $<0.0001$ \\
\hline \multicolumn{4}{|l|}{ Habit of exercise } \\
\hline No & $11,781(82.67 \%)$ & Ref & \\
\hline Yes & 2470 (17.33\%) & $-0.03(-0.04,-0.01)$ & 0.0008 \\
\hline ALT & $19.76 \pm 14.47$ & $0.01(0.01,0.01)$ & $<0.0001$ \\
\hline AST & $18.23 \pm 8.67$ & $0.01(0.01,0.01)$ & $<0.0001$ \\
\hline GGT & $19.13 \pm 16.13$ & $0.01(0.01,0.01)$ & $<0.0001$ \\
\hline $\mathrm{HDL}-\mathrm{C}$ & $1.46 \pm 0.40$ & $-0.54(-0.55,-0.52)$ & $<0.0001$ \\
\hline $\mathrm{TC}$ & $5.12 \pm 0.87$ & $0.09(0.09,0.10)$ & $<0.0001$ \\
\hline TG & $0.89 \pm 0.63$ & $0.56(0.56,0.56)$ & $<0.0001$ \\
\hline $\mathrm{TG} / \mathrm{HDL}-\mathrm{C}$ ratio & $0.73 \pm 0.74$ & $0.51(0.51,0.51)$ & $<0.0001$ \\
\hline $\mathrm{FPG}$ & $5.15 \pm 0.41$ & $0.26(0.25,0.28)$ & $<0.0001$ \\
\hline $\mathrm{HbA1c}$ & $5.18 \pm 0.32$ & $0.12(0.10,0.14)$ & $<0.0001$ \\
\hline \multicolumn{4}{|l|}{ Drinking status } \\
\hline Nor or less & 11,805 (82.84\%) & Ref & \\
\hline Light & $1758(12.34 \%)$ & $0.06(0.04,0.08)$ & $<0.0001$ \\
\hline Moderate & $688(4.83 \%)$ & $0.11(0.08,0.14)$ & $<0.0001$ \\
\hline \multicolumn{4}{|l|}{ Smoking status } \\
\hline Nor & 8746 (61.37\%) & Ref & \\
\hline Past & $2559(17.96 \%)$ & $0.14(0.12,0.15)$ & $<0.0001$ \\
\hline Current & 2946 (20.67\%) & $0.22(0.21,0.24)$ & $<0.0001$ \\
\hline SBP & $113.93 \pm 14.82$ & $0.01(0.01,0.01)$ & $<0.0001$ \\
\hline DBP & $71.12 \pm 10.38$ & $0.01(0.01,0.01)$ & $<0.0001$ \\
\hline
\end{tabular}

OR, odds ratios; other abbreviations as in Table 1

the curve of NAFLD. The results showed that among these parameters, the accuracy of $\mathrm{WC}$ in determining NAFLD was the highest, followed by CMI, and the best cutoff value of CMI in determining NAFLD was 0.3241 (Table 6). In order to further verify whether the cutoff value used by CMI to determine NAFLD was stable, we continue to use restricted cubic splines to simulate the association between CMI and NAFLD. As shown in Fig. 1, when CMI was about 0.35 , it may be a potential threshold point for NAFLD risk.

\section{Discussion}

In this cross-sectional study based on the general population, we examined the association between CMI and NAFLD, and found a strong positive correlation between
Table 3 Univariate analysis of the association between NAFLD and baseline variables

\begin{tabular}{|c|c|c|}
\hline & OR $(95 \% \mathrm{Cl})$ & $P$-value \\
\hline \multicolumn{3}{|l|}{ Sex } \\
\hline Women & Ref & \\
\hline Men & $5.02(4.51,5.58)$ & $<0.0001$ \\
\hline Age & $1.02(1.01,1.02)$ & $<0.0001$ \\
\hline Weight & $1.13(1.12,1.14)$ & $<0.0001$ \\
\hline Height & $1.06(1.05,1.06)$ & $<0.0001$ \\
\hline BMl & $1.65(1.61,1.68)$ & $<0.0001$ \\
\hline WC & $1.20(1.19,1.21)$ & $<0.0001$ \\
\hline WHtR (Per SD) & $4.06(3.82 ., 4.31)$ & $<0.0001$ \\
\hline \multicolumn{3}{|c|}{ Habit of exercise } \\
\hline No & Ref & \\
\hline Yes & $0.82(0.72,0.92)$ & 0.0008 \\
\hline ALT & $1.10(1.10,1.11)$ & $<0.0001$ \\
\hline AST & $1.09(1.08,1.10)$ & $<0.0001$ \\
\hline GGT & $1.04(1.04,1.04)$ & $<0.0001$ \\
\hline $\mathrm{HDL}-\mathrm{C}$ & $0.05(0.05,0.06)$ & $<0.0001$ \\
\hline TC & $1.65(1.57,1.73)$ & $<0.0001$ \\
\hline $\mathrm{TG}$ & $4.65(4.30,5.03)$ & $<0.0001$ \\
\hline TG/HDL-C & $3.92(3.65,4.21)$ & $<0.0001$ \\
\hline FPG & $6.91(6.13,7.78)$ & $<0.0001$ \\
\hline $\mathrm{HbA1c}$ & $4.42(3.84,5.08)$ & $<0.0001$ \\
\hline \multicolumn{3}{|l|}{ Drinking status } \\
\hline Nor or less & Ref & \\
\hline Light & $0.90(0.79,1.04)$ & 0.1442 \\
\hline Moderate & $1.12(0.92,1.36)$ & 0.2731 \\
\hline \multicolumn{3}{|l|}{ Smoking status } \\
\hline Nor & Ref & \\
\hline Past & $2.12(1.91,2.37)$ & $<0.0001$ \\
\hline Current & $1.93(1.73,2.14)$ & $<0.0001$ \\
\hline SBP & $1.05(1.05,1.06)$ & $<0.0001$ \\
\hline DBP & $1.08(1.07,1.08)$ & $<0.0001$ \\
\hline CMI & $19.64(16.97,22.73)$ & $<0.0001$ \\
\hline CMI Z score & $3.08(2.91,3.25)$ & $<0.0001$ \\
\hline
\end{tabular}

OR, odds ratios; other abbreviations as in Table 1

higher CMI and the risk of NAFLD, independent of metabolic factors such as blood glucose and lipids. As far as we know, this study provides the first evidence of the relationship between CMI and NAFLD. The findings of this study provide new ideas for the risk assessment of NAFLD and are of great significance.

NAFLD is characterized by steatosis of the liver, which promotes the occurrence and progression of NAFLD with the participation of inflammation, oxidative stress and IR $[11,32]$. In many previous observational studies, various lipid abnormalities have received widespread attention because of their significant increase in the risk of NAFLD [11,33], among which 
Table 4 Association between CMI and NAFLD in different models

\begin{tabular}{lllllll}
\hline & OR $(\mathbf{9 5} \% \mathbf{C l})$ & & & & Q \\
\cline { 2 - 6 } & Multivariable Analysis & Q1 & Q2 & Q3 & Q4 & \\
\hline Model one & $1.67(1.58,1.77)$ & Ref & $2.60(1.86,3.63)$ & $5.09(3.70,7.00)$ & $11.05(8.03,15.22)$ & $<0.0001$ \\
Model two & $1.28(1.20,1.37)$ & Ref & $2.03(1.44,2.86)$ & $3.24(2.29,4.57)$ & $5.53(3.80,8.04)$ & $<0.0001$ \\
Model three & $1.28(1.19,1.37)$ & Ref & $2.11(1.49,3.01)$ & $3.48(2.44,4.96)$ & $5.55(3.77,8.19)$ & $<0.0001$ \\
\hline
\end{tabular}

OR: odds ratios; CMI: cardiometabolic index; NAFLD: non-alcoholic fatty liver disease

Model one adjusted for: sex, age, BMI, drinking status and smoking status

Model two adjusted for: sex, BMI, WHtR, GGT, HDL-C, TC, FPG, drinking status and SBP

Model three adjusted for: sex, age, BMI, ALT, AST, height, WHtR, GGT, habit of exercise, HDL-C, TC, FPG, drinking status, smoking status, SBP and HbA1C

Table 5 Stratified associations between CMI and NAFLD by age, sex, BMI, and habit of exercise

\begin{tabular}{|c|c|c|c|c|}
\hline Subgroup & No. of participants & Unadjusted OR (95\% Cl) & Adjusted OR $(95 \% \mathrm{Cl})$ & $P$ for interaction \\
\hline Age (years) & & & & 0.0010 \\
\hline $18-30$ & 645 & $9.72(5.49,17.21)$ & $2.63(1.45,4.78)$ & \\
\hline $31-45$ & 8204 & $3.75(3.47,4.07)$ & $1.38(1.27,1.51)$ & \\
\hline $46-60$ & 4906 & $2.42(2.23,2.62)$ & $1.18(1.09,1.29)$ & \\
\hline$>60$ & 487 & $1.66(1.35,2.04)$ & $1.14(0.93,1.40)$ & \\
\hline Sex & & & & 0.0045 \\
\hline Women & 6840 & $5.23(4.50,6.07)$ & $1.58(1.34,1.87)$ & \\
\hline Men & 7402 & $2.26(2.13,2.39)$ & $1.26(1.17,1.35)$ & \\
\hline BMI $\left(\mathrm{kg} / \mathrm{m}^{2}\right)$ & & & & 0.1705 \\
\hline$<18.5$ & 1543 & $4.51(0.80,25.27)$ & $1.83(0.21,16.01)$ & \\
\hline$\geq 18.5,<24$ & 9334 & $2.37(2.19,2.57)$ & $1.33(1.22,1.46)$ & \\
\hline$\geq 24,<28$ & 2721 & $1.75(1.61,1.89)$ & $1.22(1.12,1.32)$ & \\
\hline$\geq 28$ & 644 & $2.26(1.78,2.87)$ & $1.50(1.19,1.89)$ & \\
\hline Habit of exercise & & & & 0.0851 \\
\hline Yes & 2468 & $3.19(3.00,3.39)$ & $1.31(1.22,1.41)$ & \\
\hline No & 11,774 & $2.54(2.22,2.89)$ & $1.18(1.05,1.32)$ & \\
\hline
\end{tabular}

OR: odds ratios; other abbreviations as in Table 1

Adjusted for: sex, BMI, WHtR, GGT, HDL-C, TC, FPG, drinking status and SBP

Table 6 The AUC for each index to discriminate NAFLD

\begin{tabular}{|c|c|c|c|c|c|c|}
\hline & AUC & $95 \% \mathrm{Cl}$ low & $95 \% \mathrm{Cl}$ upp & Cutoff value & Specificity & Sensitivity \\
\hline $\mathrm{CMl}$ & 0.8359 & 0.8279 & 0.8439 & 0.3241 & 0.7409 & 0.7787 \\
\hline WC & 0.8610 & 0.8539 & 0.8681 & 79.6500 & 0.7571 & 0.8085 \\
\hline Height & 0.6395 & 0.6280 & 0.6510 & 164.1500 & 0.5244 & 0.7164 \\
\hline TG & 0.7969 & 0.7877 & 0.8061 & 0.8411 & 0.6799 & 0.7698 \\
\hline $\mathrm{HDL}-\mathrm{C}$ & 0.7587 & 0.7489 & 0.7685 & 1.3408 & 0.6481 & 0.7543 \\
\hline
\end{tabular}

AUC, area under the curve; $\mathrm{Cl}$, confidence interval; HDL-C, high-density lipoprotein cholesterol; LDL-C, low-density lipoprotein cholesterol; NAFLD, non-alcoholic fatty liver disease; WC, Waist circumference; TG, triglyceride; CMI, cardiometabolic index

the disorder of lipoproteins rich in TG is considered to be a key factor in cardiovascular risk in patients with NAFLD [34]. In this context, various lipid parameters and lipid ratios have been widely explored and studied, among which TG/HDL-C ratio seems to be a better predictor of NAFLD [13, 14], which may be related to IR. Several recent studies have shown that TG/HDL-C ratio is significantly associated with IR in whites and yellow race, and is considered to be an effective clinical alternative marker for IR $[15,35,36]$, while it seems 


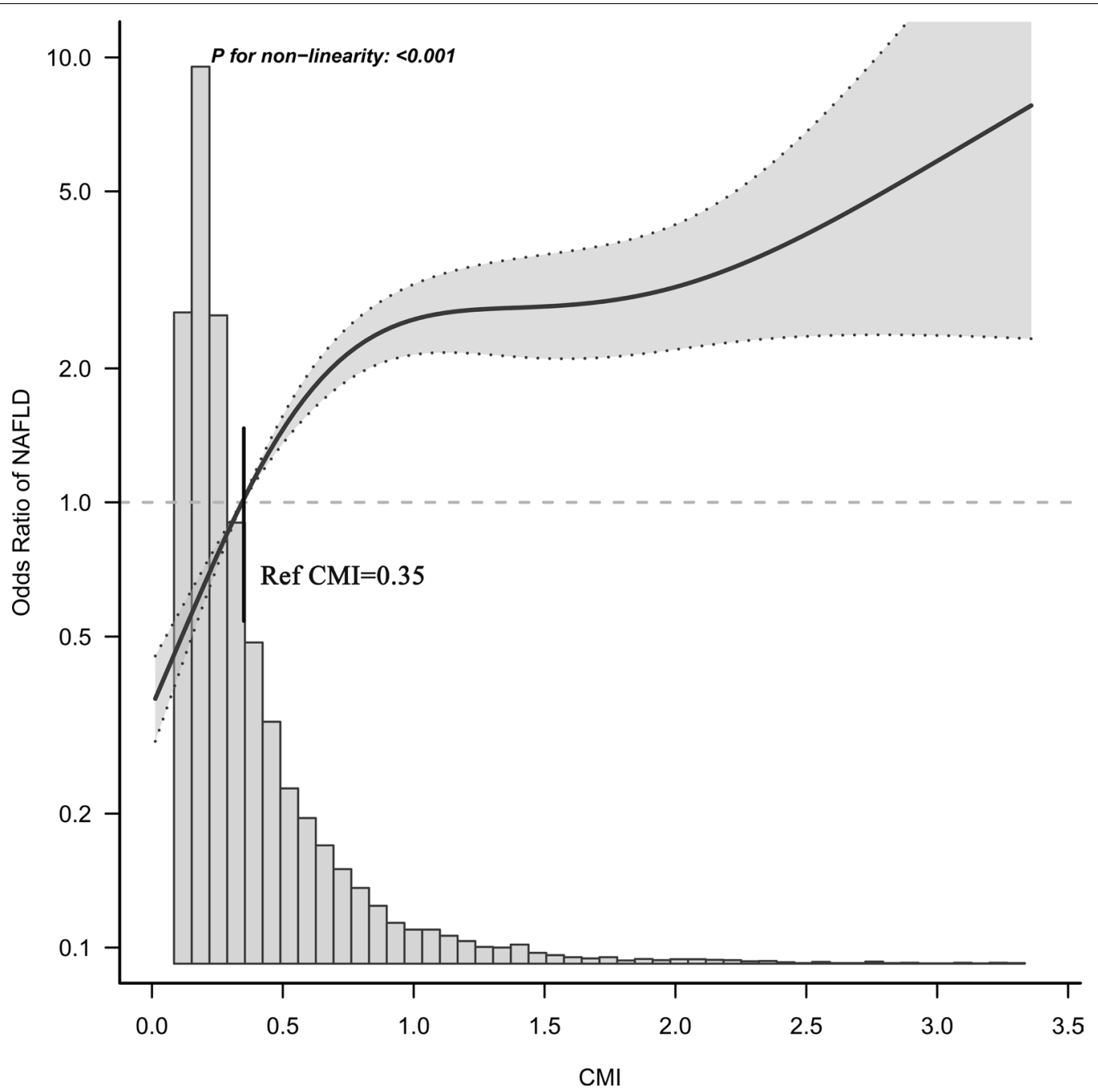

Fig. 1 Odds ratios of NAFLD based on CMI. Model was adjusted for sex, age, BMI, ALT, AST, height, WHtR, GGT, habit of exercise, HDL-C, TC, FPG, drinking status, smoking status, SBP and HbA1c. Data were fitted by a restricted cubic spline logistic regression model. The $95 \%$ confidence intervals are indicated by the dashed line

inappropriate to use TG/HDL-C ratio to predict IR in Hispanics and African Americans [15]. At present, drugs for the treatment of NAFLD with dyslipidemia as the entry point are also being extensively studied [11, 37, 38].

Obesity is the main risk factor for the prompt increase of NAFLD in recent years, and most obesityrelated parameters are also important risk factors for NAFLD [12, 39]. Both BMI and WC are the most commonly used anthropometric indicators to evaluate obesity. Their measurements are simple, convenient and reproducible, so they have been widely used in the clinic. However, BMI has an obvious limitation, it may not be able to distinguish excess adipose tissue [40,
41], while WC is an excellent indicator of visceral obesity. In recent years, more and more studies have found that WHtR, which combines height and WC, is a better alternative indicator of obesity, and it is also a better determinant of NAFLD risk than BMI and WC $[16,19]$.

$\mathrm{CMI}$ is a newly developed clinical indicator that is a marker of the combination of WHtR and TG/HDL-C ratio. It was first reported by Wakabayashi et al. in 2015 that it has a significant advantage in the evaluation of diabetes [20]. Subsequent studies have further found that CMI is closely associated with hypertension, stroke, cardiovascular disease, and kidney disease [2225], suggesting that CMI has value as an indicator of metabolic diseases. However, there is currently no data 
on the association between CMI and NAFLD. In this sense, this study verified the correlation between CMI and NAFLD in the general population for the first time based on a large sample size.

In the subgroup analysis, the study also assessed whether there were differences in the association between CMI and NAFLD in different populations. The result showed that there were significant differences between CMI and NAFLD in different ages and genders, but no significant statistical differences were found in BMI and habit of exercise stratification. Notably, women were found to have a higher risk of CMI-related NAFLD than men in this study, while similar results appear to have been found in several other CMI-related studies [20, $21,23,24]$. In an earlier study by Wakabayashi et al., they evaluated 10,196 subjects who took a health check and found that the association between CMI and diabetes was stronger in women than in men (OR: 14.61 for women, 5.38 for men) [20]. Sun and his team also found this gender difference in the Chinese population in a follow-up study [21]. And in 2017, Wang and his colleagues found that CMI also differed between the sexes in cerebrovascular disease and hypertension, with CMI significantly more strongly associated with stroke and hypertension in women than in men $[23,24]$. Taken together, CMI seems to be a more suitable marker for metabolic-related diseases in women. In addition, this study also found that among young people, the risk of CMI-related NAFLD was significantly higher than that of other age groups. In order to explain this special phenomenon, we further describe the baseline variables according to age (Additional file 1: Table S2), and it is worth noting that in this study, young NAFLD patients have higher BMI, WC, TG/ HDL-C ratio and CMI values, while relatively few people maintain exercise habits. With the current obesity epidemic, young people in today's society may already be overweight or obese in childhood [3, 42]. Furthermore, with the accelerated development of social information, the aggravation of social aging, and accompanied by a wide range of unhealthy living habits among young people, young people also begin to suffer from NAFLD and other metabolic diseases prematurely [43-45]. The results of this study suggest that young people should enhance physical exercise to avoid obesity.

\section{Research strengths and limitations}

The advantage of this study will be reflected in that it conducted an epidemiological survey of the general population in the case of large sample size, and subgroup analysis was used to check whether there were differences between CMI and NAFLD in different populations. As far as we know, this study reports the data of the relationship between CMI and NAFLD for the first time.
This study also has some limitations. First, the diagnosis of NAFLD in this study was evaluated by abdominal ultrasound; it is undeniable that abdominal ultrasound may not be sensitive enough for mild fatty liver [46]. However, for large-scale epidemiological investigations, liver biopsy does not seem to be a suitable choice, which is an urgent problem to be solved at present. Second, the current study adopts a cross-sectional design, so it is impossible to explain whether there is a causality relationship between CMI and NAFLD. Third, due to the lack of some parameters for calculating the score of liver fibrosis in this study, it is impossible to analyze the association between CMI and liver fibrosis in the current study. Fourth, because subjects with diabetes and impaired fasting blood glucose were excluded from the study, there may be a certain selection bias. Finally, although the current research has adopted strict standards to identify confounding factors, there are still some potential residual confounding variables because they are unmeasurable or not collected in this data set.

\section{Conclusion}

Current studies have shown that there is a strong positive correlation between higher CMI and the risk of NAFLD in the general population, independent of traditional risk factors. Additionally, the results of subgroup analysis showed that the CMI-related NAFLD risk of young people was significantly higher than that of other age groups, and the CMI-related NAFLD risk of women was significantly higher than that of men. The content of this study suggests that regular examination and evaluation of CMI may help control the occurrence of NAFLD, and young people should strengthen physical exercise and pay attention to body obesity.

\section{Abbreviations \\ CMI: Cardiometabolic index; NAFLD: Non-alcoholic fatty liver disease; SD: Standard deviation; TG/HDL-C: Triglyceride/high-density lipoprotein choles- terol; IR: Insulin resistance; WHtR: Waist-to-height ratio; WC: Waist circumfer- ence; ALT: Alanine aminotransferase; AST: Aspartate aminotransferase; GGT : Gamma-glutamyl transferase; TC: Total cholesterol; HbA1c: Hemoglobin A1c; FPG: Fasting blood glucose; OR: Odds ratio; Cl: Confidence interval; BMI: Body mass index; S/DBP: Systolic/diastolic blood pressure.}

\section{Supplementary Information}

The online version contains supplementary material available at https://doi. org/10.1186/s12876-022-02099-y.

Additional file 1. Supplementary Table 1: Collinearity diagnostics steps.

Acknowledgements

Thanks to all the researchers and subjects who participated in the study. 


\section{Authors' contributions}

Study concept and design: JW-Z and YZ. Study conduct: JW-Z, HL-X, HM-Z, $\mathrm{CH}, \mathrm{SL}$ and $Y Z$. Data analysis: JW-Z nad YZ. Writing of the first draft: JW-Z, HL-X, $\mathrm{HM}-\mathrm{Z}, \mathrm{CH}$ and SL. Review and revision of the article: All authors. All authors read and approved the final manuscript.

\section{Funding}

No.

\section{Availability of data and materials}

The datasets analyzed during the current study are available in the [DRYAD] repository (https://datadryad.org/stash/dataset/doi:10.5061\%2Fdryad.8q0p1 92).

\section{Declarations}

\section{Ethics approval and consent to participate}

Not applicable. This study was a post hoc analysis of the NAGALA cohort, and the data were obtained from the Dryad database. We confirm that all the methods used in this study were performed in accordance with the relevant guidelines and regulations.

\section{Competing interests}

The authors declare that they have no competing interests.

\section{Consent for publication}

Not applicable. This study was a post hoc analysis of the NAGALA cohort, and the submitted materials did not provide any identifying information about the participants.

\section{Author details}

'Department of Cardiology, Jiujiang First People's Hospital, Jiujiang 332000, China. ${ }^{2}$ Department of Cardiology, Jiangxi Provincial People's Hospital, Nanchang 330006, China. ${ }^{3}$ Gastroenterology Department, Jiangxi Provincial People's Hospital, Nanchang 330006, China. ${ }^{4}$ Jiangxi Cardiovascular Research Institute, Jiangxi Provincial People's Hospital, Nanchang 330006, China.

Received: 3 August 2021 Accepted: 6 January 2022

Published online: 12 January 2022

\section{References}

1. Younossi ZM. Non-alcoholic fatty liver disease: a global public health perspective. J Hepatol. 2019;70:531-44.

2. Zhang L, She ZG, Li H, Zhang XJ. Non-alcoholic fatty liver disease: a metabolic burden promoting atherosclerosis. Clin Sci (Lond). 2020;134:1775-99.

3. Younossi ZM, Koenig AB, Abdelatif D, Fazel Y, Henry L, Wymer M. Global epidemiology of nonalcoholic fatty liver disease-Meta-analytic assessment of prevalence, incidence, and outcomes. Hepatology. 2016;64:73-84.

4. Fazel Y, Koenig AB, Sayiner M, Goodman ZD, Younossi ZM. Epidemiology and natural history of non-alcoholic fatty liver disease. Metabolism. 2016;65:1017-25.

5. Estes C, Anstee QM, Arias-Loste MT, Bantel H, Bellentani S, Caballeria $J$, et al. Modeling NAFLD disease burden in China, France, Germany, Italy, Japan, Spain, United Kingdom, and United States for the period 2016-2030. J Hepatol. 2018;69:896-904.

6. Estes C, Razavi H, Loomba R, Younossi Z, Sanyal AJ. Modeling the epidemic of nonalcoholic fatty liver disease demonstrates an exponential increase in burden of disease. Hepatology. 2018;67:123-33.

7. McPherson S, Hardy T, Henderson E, Burt AD, Day CP, Anstee QM. Evidence of NAFLD progression from steatosis to fibrosing-steatohepatitis using paired biopsies: implications for prognosis and clinical management. J Hepatol. 2015;62:1148-55.

8. Mikolasevic I, Milic S, Turk Wensveen T, Grgic I, Jakopcic I, Stimac D, et al. Nonalcoholic fatty liver disease: a multisystem disease? World J Gastroenterol. 2016;22:9488-505.
9. Byrne CD, Targher G. NAFLD: a multisystem disease. J Hepatol. 2015;62(Suppl 1):S47-64.

10. Long MT, Wang N, Larson MG, Mitchell GF, Palmisano J, Vasan RS, et al Nonalcoholic fatty liver disease and vascular function: cross-sectional analysis in the Framingham heart study. Arterioscler Thromb Vasc Biol. 2015;35:1284-91.

11. Katsiki N, Mikhailidis DP, Mantzoros CS. Non-alcoholic fatty liver disease and dyslipidemia: an update. Metabolism. 2016;65:1109-23.

12. Polyzos SA, Kountouras J, Mantzoros CS. Obesity and nonalcoholic fatty liver disease: From pathophysiology to therapeutics. Metabolism. 2019;92:82-97.

13. Fan N, Peng L, Xia Z, Zhang L, Song Z, Wang Y, et al. Triglycerides to highdensity lipoprotein cholesterol ratio as a surrogate for nonalcoholic fatty liver disease: a cross-sectional study. Lipids Health Dis. 2019;18:39.

14. Fukuda Y, Hashimoto Y, Hamaguchi M, Fukuda T, Nakamura N, Ohbora A, et al. Triglycerides to high-density lipoprotein cholesterol ratio is an independent predictor of incident fatty liver; a population-based cohort study. Liver Int. 2016;36:713-20.

15. Kim-Dorner SJ, Deuster PA, Zeno SA, Remaley AT, Poth M. Should triglycerides and the triglycerides to high-density lipoprotein cholesterol ratio be used as surrogates for insulin resistance? Metabolism. 2010;59:299-304

16. Tomas Ž, Škarić-Jurić T, Zajc Petranović M, Jalšovec M, Rajić Šikanjić P, Smolej NN. Waist to height ratio is the anthropometric index that most appropriately mirrors the lifestyle and psychological risk factors of obesity. Nutr Diet. 2019;76:539-45.

17. Ashwell M, Gunn P, Gibson S. Waist-to-height ratio is a better screening tool than waist circumference and BMI for adult cardiometabolic risk factors: systematic review and meta-analysis. Obes Rev. 2012;13:275-86.

18. Corrêa MM, Thumé E, De Oliveira ER, Tomasi E. Performance of the waistto-height ratio in identifying obesity and predicting non-communicable diseases in the elderly population: a systematic literature review. Arch Gerontol Geriatr. 2016:65:174-82.

19. Sheng G, Xie Q, Wang R, Hu C, Zhong M, Zou Y. Waist-to-height ratio and non-alcoholic fatty liver disease in adults. BMC Gastroenterol. 2021:21:239.

20. Wakabayashi I, Daimon T. The, "cardiometabolic index" as a new marker determined by adiposity and blood lipids for discrimination of diabetes mellitus. Clin Chim Acta. 2015;438:274-8.

21. Shi WR, Wang HY, Chen S, Guo XF, Li Z, Sun YX. Estimate of prevalent diabetes from cardiometabolic index in general Chinese population: a community-based study. Lipids Health Dis. 2018;17:236.

22. Dursun M, Besiroglu H, Otunctemur A, Ozbek E. Association between cardiometabolic index and erectile dysfunction: a new index for predicting cardiovascular disease. Kaohsiung J Med Sci. 2016;32:620-3.

23. Wang H, Chen Y, Guo X, Chang Y, Sun Y. Usefulness of cardiometabolic index for the estimation of ischemic stroke risk among general population in rural China. Postgrad Med. 2017;129:834-41.

24. Wang H, Chen Y, Sun G, Jia P, Qian H, Sun Y. Validity of cardiometabolic index, lipid accumulation product, and body adiposity index in predicting the risk of hypertension in Chinese population. Postgrad Med. 2018;130:325-33.

25. Wang HY, Shi WR, Yi X, Wang SZ, Luan SY, Sun YX. Value of reduced glomerular filtration rate assessment with cardiometabolic index: insights from a population-based Chinese cohort. BMC Nephrol. 2018;19:294.

26. Okamura T, Hashimoto Y, Hamaguchi M, Obora A, Kojima T, Fukui M. Ectopic fat obesity presents the greatest risk for incident type 2 diabetes: a population-based longitudinal study. Int J Obes (Lond). 2019;43:139-48.

27. Okamura T, et al. (2019), Data from: ectopic fat obesity presents the greatest risk for incident type 2 diabetes: a population-based longitudinal study, Dryad, Dataset.https://doi.org/10.5061/dryad.8q0p192.

28. Choi JH, Sohn W, Cho YK. The effect of moderate alcohol drinking in nonalcoholic fatty liver disease. Clin Mol Hepatol. 2020;26:662-9.

29. Hamaguchi M, Kojima T, Itoh Y, Harano Y, Fujii K, Nakajima T, et al. The severity of ultrasonographic findings in nonalcoholic fatty liver disease reflects the metabolic syndrome and visceral fat accumulation. Am J Gastroenterol. 2007;102:2708-15.

30. Fitchett EJA, Seale AC, Vergnano S, Sharland M, Heath PT, Saha SK, et al. Strengthening the Reporting of Observational Studies in Epidemiology for Newborn Infection (STROBE-NI): an extension of the STROBE statement for neonatal infection research. Lancet Infect Dis. 2016;16:e202-13. 
31. Vandenbroucke JP, von Elm E, Altman DG, Gøtzsche PC, Mulrow CD, Pocock SJ, et al. Strengthening the reporting of observational studies in epidemiology (STROBE): explanation and elaboration. Int J Surg. 2014;12:1500-24.

32. Ipsen DH, Lykkesfeldt J, Tveden-Nyborg P. Molecular mechanisms of hepatic lipid accumulation in non-alcoholic fatty liver disease. Cell Mol Life Sci. 2018;75:3313-27.

33. Zou Y, Zhong L, Hu C, Zhong M, Peng N, Sheng G. LDL/HDL cholesterol ratio is associated with new-onset NAFLD in Chinese non-obese people with normal lipids: a 5-year longitudinal cohort study. Lipids Health Dis. 2021;20:28.

34. Zou Y, Lan J, Zhong Y, Yang S, Zhang H, Xie G. Association of remnant cholesterol with nonalcoholic fatty liver disease: a general population-based study. Lipids Health Dis. 2021;20:139.

35. Yang Y, Wang B, Yuan H, Li X. Triglycerides to high-density lipoprotein cholesterol ratio is the best surrogate marker for insulin resistance in nonobese middle-aged and elderly population: a cross-sectional study. Int J Endocrinol. 2021;2021:6676569.

36. Du T, Yuan G, Zhang M, Zhou X, Sun X, Yu X. Clinical usefulness of lipid ratios, visceral adiposity indicators, and the triglycerides and glucose index as risk markers of insulin resistance. Cardiovasc Diabetol. 2014;13:146.

37. Pastori D, Pani A, Di Rocco A, Menichelli D, Gazzaniga G, Farcomeni A, et al. Statin liver safety in non-alcoholic fatty liver disease: a systematic review and metanalysis. Br J Clin Pharmacol. 2021. https://doi.org/10. 1111/bcp.14943.

38. Scorletti E, Byrne CD. Omega-3 fatty acids and non-alcoholic fatty liver disease: Evidence of efficacy and mechanism of action. Mol Aspects Med. 2018;64:135-46.

39. Sheng G, Lu S, Xie Q, Peng N, Kuang M, Zou Y. The usefulness of obesity and lipid-related indices to predict the presence of non-alcoholic fatty liver disease. Lipids Health Dis. 2021;20:134.

40. Kyle UG, Schutz Y, Dupertuis YM, Pichard C. Body composition interpretation. Contributions of the fat-free mass index and the body fat mass index. Nutrition. 2003;19:597-604.

41. Zou Y, Sheng G, Yu M, Xie G. The association between triglycerides and ectopic fat obesity: an inverted U-shaped curve. PLOS ONE. 2020;15:e0243068.

42. Cuthbertson DJ, Brown E, Koskinen J, Magnussen CG, Hutri-Kähönen $\mathrm{N}$, Sabin M, et al. Longitudinal analysis of risk of non-alcoholic fatty liver disease in adulthood. Liver Int. 2019;39:1147-54.

43. Kennedy BK, Partridge L. 2nd interventions in aging conference. Aging (Albany NY). 2017;9:1090-5.

44. American College of Obstetricians and Gynecologists' Committee on Adolescent Health Care. Committee Opinion No. 653: concerns regarding social media and health issues in adolescents and young adults. Obstet Gynecol. 2016;127:e62-5.

45. McMahon DM, Burch JB, Youngstedt SD, Wirth MD, Hardin JW, Hurley TG, et al. Relationships between chronotype, social jetlag, sleep, obesity and blood pressure in healthy young adults. Chronobiol Int. 2019;36:493-509.

46. Ferraioli G, Soares Monteiro LB. Ultrasound-based techniques for the diagnosis of liver steatosis. World J Gastroenterol. 2019;25:6053-62.

\section{Publisher's Note}

Springer Nature remains neutral with regard to jurisdictional claims in published maps and institutional affiliations.

Ready to submit your research? Choose BMC and benefit from:

- fast, convenient online submission

- thorough peer review by experienced researchers in your field

- rapid publication on acceptance

- support for research data, including large and complex data types

- gold Open Access which fosters wider collaboration and increased citations

- maximum visibility for your research: over $100 \mathrm{M}$ website views per year

At BMC, research is always in progress.

Learn more biomedcentral.com/submissions 